\section{Upper Gastrointestinal Bleeding Due to Metastatic Gastric and Duodenal Squamous-Cell Carcinoma from the Hypopharynx}

The gastrointestinal tract is rarely the site of a metastatic neoplasm, and the literature does not to our knowledge contain any references to gastrointestinal metastasis from a hypopharyngeal squamous-cell carcinoma. We report here on a case of hypopharyngeal carcinoma with metastasis to the stomach and duodenum, resulting in frank upper gastrointestinal bleeding.

A 68-year-old man was admitted in May 1991, his major symptoms being mild dysphagia and the presence of a bulging mass on the left upper neck over the previous three months. A biopsy of the mass showed it to be a hypopharyngeal squamous-cell carcinoma. The patient received debulking surgery and radiotherapy. Two years later, he presented again, suffering from hematemesis. Panendoscopy disclosed a polypoid mass with a ragged surface and irregular ulcerations on the greater curvature side of the middle gastric body (Figure 1). Another polypoid mass with a wide base and eroded surface was found in the second portion of the duodenum. The pathology revealed a metastatic squamous-cell carcinoma of the stomach (Figure 2), which was histologically similar to the earlier hypopharyngeal carcinoma. In spite of blood transfusion and hemostatic treatment, intermittent bleeding persisted. The patient ultimately died due to aspiration pneumonia and multiple organ failure.

Metastasis to the stomach from malignant tumors arising elsewhere is generally rare. Malignant melanoma, breast cancer, lung cancer, and pancreatic cancer are the most common forms of primary cancer that lead to gastric metastasis. In the report by MacDonald, only four of 4725 patients with gastric metastases had had primary head and neck cancers (1). The clinical presentation of gastric metastasis is asymptomatic or nonspecific, with symptoms such as anorexia, nausea, vomiting, and weight loss (2). Slight blood loss and iron-deficiency anemia may be found in some cases, but frank upper gastrointestinal bleeding has not previously been seen. The most effective treatment for a bleeding metastatic lesion is surgical intervention (3). However, the patient refused more advanced treatment, including surgery.

C.S. Wu', C. M. Chen', S. Y. Tung', K. Y. Chang', T. C. Chen'

Dept. of Hepatogastroenterology

${ }^{2}$ Dept. of Pathology, Chang Gung Memorial Hospital, Taipei, Taiwan

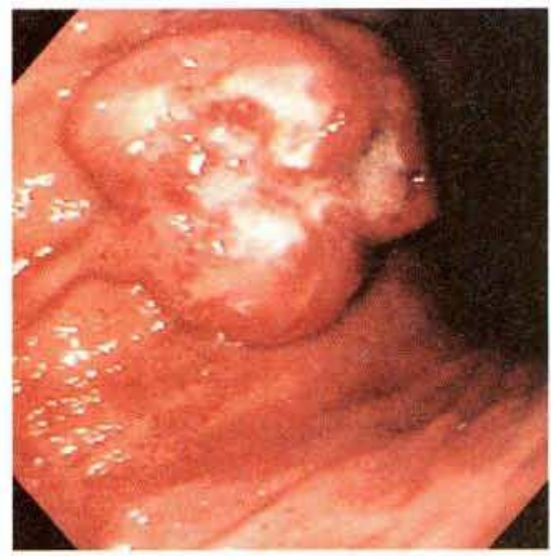

Figure 1: A polypoid fungating mass in the stomach.

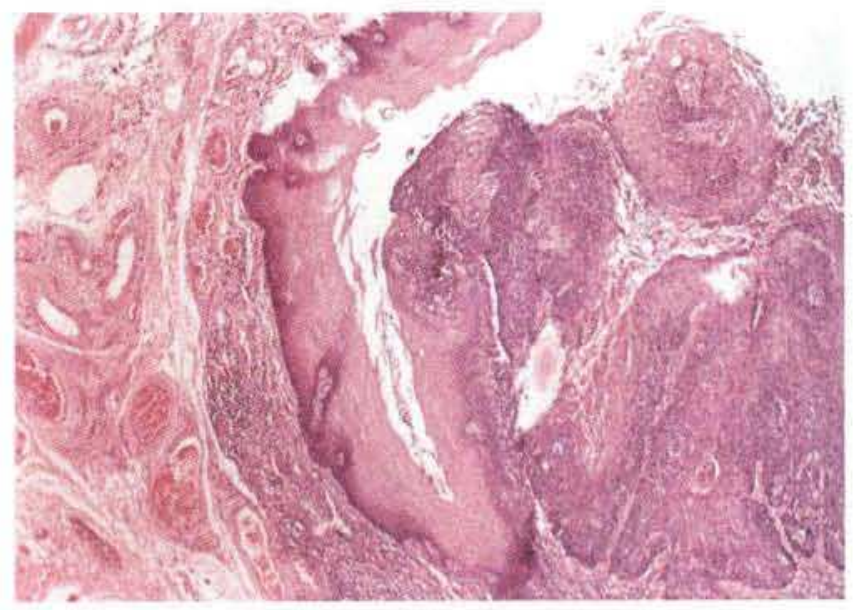

Figure 2: Metastatic squamous-cell carcinoma in the stomach (hematoxylin-eosin, $\times 200$ ).

\section{References}

1. MacDonald DJ. Multiple primaries. In: Cancer of the gastrointestinal tract. Chicago: Yearbook Medical, 1967: 269-82.

2. Menuck LS, Amberg JR. Metastatic disease involving the stomach. Am J Dig Dis 1974; 20: 903-13.

3. Gateley CA, Lewis WG, Sturdy DE. Massive lower gastrointestinal haemorrhage secondary to metastatic squamous cell carcinoma of the lung. Br J Clin Pract 1993; 47: 276-7.

\author{
Corresponding Author \\ Cheng-Shyong Wu MD \\ Gastroenterology Department \\ Chang Gung Memorial Hospital \\ 199, Tung Hwa North Road \\ Taipei, Taiwan 10591 \\ Fax: $+886-3-3272236$
}

